\title{
1 Prospects of raising baobab (Adansonia digitata L.) to fruiting in two years
}

2

3 Kenneth Fafa Egbadzor* and Jones Akuaku

4

5 Department of Agro Enterprise Development, Faculty of Applied Science and Technology, Ho

6 Technical University, Ho, Ghana.

7

8

* Corresponding author

9 kegbadzor@htu.edu.gh

10

11 Both authors contributed equally to the work.

12

13

14

15

16

17

18

19

20

21

22

23

24

25

26

27

28

29 


\section{Prospects of raising baobab (Adansonia digitata L.) to fruiting in two years}

31

32

33

34

35

36

37

38

39

40

41

42

43

44

45

46

\section{$47 \quad$ Key Words}

48

49

50

51

52

53

54

Abstract

0

1

2

3

4

Baobab is a very important plant with several uses, serving as food security crop and has the potential to raise income of farmers. However, the plant is undomesticated. We have the objective of domesticating the plant and promote its cultivation and utilization. Seeds were treated with sulphuric acid for early germination and grafted with matured scions suspected to be fruiting for several years. Twenty-two of the seedlings were transplanted on the field at Ho Technical University campus. Unfortunately, only four survived bushfire and destruction by stray animals. Surprisingly, one of the four surviving trees anthesis within twenty-eight months after seed treatment (twenty months after transplanting). This is the earliest ever reported anthesis of baobab. With better agronomic practices such as supplementary irrigation, insect pest control, manuring and protection from stray animals, baobab could start fruiting within two years of planting. This would alleviate fear of long maturity period and motivate farmers to go into baobab plantation. This would help diversify agriculture in many African countries, increase income and food security and contribute towards the attainment of the sustainable development goals of the United Nations.

\section{Anthesis, Domestication, Grafting, Scarification, Vegetative Propagation}




\section{Introduction}

56

57 Baobab (Adasonia digitata L.) domestication has been suggested by a number of authors in the past due to its importance (Jamnadass et al, 2011; Simbo et al, 2012; North et al, 2014). It is considered one of the most important African indigenous fruit trees needing domestication (Gebauer et al, 2016). This call started many years ago by Michel Adanson in whose honor the plant was scientifically named Adansonia digitata (www.powbab.com/pages/baobab-tree). There was a report on consortium of scientist working on baobab domestication in 2005 (Jensen et al, 2011). However, there are no results indicating sustained interest and work on the domestication of the plant at the moment.

In 2008, baobab was accepted as novel food in Europe and the United States of America (North et $a l, 2014)$. That might have triggered the high demand for its products in Europe and North America (Venter and Witkowski, 2010). Dependence on wild baobab products cannot meet world demand. Thus, domesticating the plant is imperative to sustainable supply to meet local and external demand. Revisiting the idea of baobab domestication is therefore, a step in the right direction.

Among the issues concerning baobab domestication and cultivation are the problem of seed dormancy and long juvenile period of the plant. Exposure to high degrees of heat in nature strongly influence germination of baobab seeds (Lautenschlager et al, 2020). Artificially, research has shown that treating the seed with concentrated sulphuric acid gives early and uniform germination. Boiling water and other treatments have also been reported to aid germination by Venter (2016) and El-Bably and Rashed (2018) among others authors.

In the case of long juvenile period, the use of vegetative planting material is most appropriate as known in other plants. Vegetative propagation methods thus need to be explored to reduce the maturity period of the plant to attract farmers to cultivate it. Vegetative propagation also results in 
78 producing true to type plants and will be particularly important in baobab to help manage wide

79 diversity that could result from seed propagation (Agbohessou et al, 2020). The vegetative

80 methods ever reported in baobab include grafting, cutting and in vitro propagation (Agbohessou

81 et al, 2020).

82 Grafting has been the mostly used among the vegetative methods. Anjarwalla et al (2017) grafted

83 scions on one to two-year-old rootstocks while Mbora et al (2008) used three months old

84 rootstocks. Graft success was high for all ages of scions used. The most important consideration

85 seems therefore to be the rootstock and scion sizes. Every available method has to be explored in

86 working towards early fruiting of baobab in order that the long maturity period would not be

87 discouraging factor in considering baobab farming. The objective of the study therefore, was to use sulphuric acid scarification for seed germination and grafting to investigate how early baobab can flower and bear fruit.

\section{Materials and Method}

\section{$91 \quad$ Seed treatment}

92 Baobab seeds were soaked in $95 \%$ sulphuric acid $\left(\mathrm{H}_{2} \mathrm{SO}_{4}\right)$ for six hours after which they were

93 removed and washed thoroughly in tap water. Washed seeds were placed on wet paper towel and

94 covered with same. Germination (protrusion of radicles) occurred five days after placing in the

95 paper towel.

\section{Nursing and nursery practices}

97 Germinating seeds were planted in soil contained in nursery bags $(20 \mathrm{~cm}$ height and $15 \mathrm{~cm}$

98 diameter). Nursery bags were placed under citrus trees that allowed only partial penetration of

99 sunlight. Watering was done whenever necessary. Pruning of the citrus tree was done after five 
100 weeks to allow more light to the baobab seedlings. There was no application of insecticides nor

101 fertilizers at the nursery stage.

102 Grafting

103 Scions were taken from matured baobab trees suspected to be fruiting for several years and grafted

104 on the seedlings (rootstock) seven months after seed treatment. Top cleft method was used in 105 grafting.

106 Transplanting and other cares

107 Transplanting was done at Ho Technical University campus in October, 2019, that was three 108 months after grafting. Apart from watering at the time of transplanting, no other irrigation was 109 done apart from natural precipitation. Two hand-trowel full of poultry manure was supplied two 110 months after transplanting. Broad spectrum insecticide was applied to control leaf eating insects.

111 Weed was regularly controlled.

112 Observations and Discussion

113 Baobab radicle emergence which was taken to be seed germination occurred five days (average)

114 after treatment with sulphuric acid. All the pregerminated seeds transferred to soil filled nursery 115 bags emerged from the soil. The seedlings grew rapidly and were grafted within seven months 116 after planting with about $90 \%$ success. Grafting at seven months is significant improvement over 117 one to two years rootstock recommended by Anjarwalla et al (2017). However, this can be further 118 reduced considering the three-month-old rootstocks reported by Mbora et al (2008). At three 119 months, the rootstocks used in this trial were too small for the available scions. Different rootstocks 120 can be explored to select faster growing ones to shorten the grafting time.

121 Growth was generally rapid during the raining seasons and very slow during the dry seasons 122 characterized by dropping of leaves. First anthesis was observed 824 days (28 months or two years, 
123 four months) after seed treatment as shown in Table 1. The period from transplanting to anthesis 124 was only 595 days (about 20 months). This observation in less than two years after transplanting 125 is an extra ordinary. Jensen et al (2011), reported anthesis in baobab to be between eight and 126 twenty-three (8-23) years when planted by seed or three to five (3-5) years when grafted. However, 127 in recent publication, Agbohessou et al 2020 stated that result of grafted baobab to fruiting has not 128 yet been documented. There are two similar but different issues here. One is flowering and the 129 other fruiting. In both cases, our observation of flowering is earlier than ever reported. Prior to the 130 current study, the earliest ever reported flowering of grafted baobab was by Sidibe and Williams 131 (2002) and Mbora et al (2008). They reported that grafted baobab can flower after three years. Our 132 observation is earlier than the three years, thus making it currently, the earliest baobab flowering 133 ever reported.

Table 1: Timeline of activities and observations

\begin{tabular}{|l|l|l|l|l|l|l|}
\hline \multirow{2}{*}{} & \multicolumn{6}{|c|}{ Activity } \\
\cline { 2 - 8 } & Seed treatment & Sowing & Grafting & Transplanting & Bud & Anthesis \\
\cline { 2 - 8 } & $17 / 03 / 19$ & $22 / 03 / 19$ & $09 / 10 / 19$ & $19 / 11 / 19$ & $21 / 06 / 21$ & $06 / 07 / 21$ \\
\hline Days P & 0 & 5 & 201 & 41 & 580 & 15 \\
\hline Days B & 0 & 5 & 206 & 247 & 827 & 842 \\
\hline
\end{tabular}

137 beginning of the investigation. matured scion and probably the good climate as well, led to the early flowering observed. With 
141 good agronomic practices and earlier grafting than we did, anthesis and fruiting could even be

142 earlier than this observation. This result could encourage farmers to grow baobab.

144 At the time of anthesis, the height of the tree was only $170 \mathrm{~cm}$ as recorded in Table 2 . The first

145 branch was as low as $30 \mathrm{~cm}$ and the circumference of tree trunk at ground level amd at first branch

$146 \quad 35.4 \mathrm{~cm}$ and $27.7 \mathrm{~cm}$ respective;y. These values are far lower than would be expected in nature.

147 Tree stature would make management easy. The tree and flower are shown in Figs. 1 and 2.

Table 2: Data on tree at first anthesis

\begin{tabular}{|l|l|l|}
\cline { 2 - 3 } 151 & Character & Measurement \\
\cline { 2 - 3 } 152 & The plant height at first flowering was & $170 \mathrm{~cm}$ \\
\cline { 2 - 3 } 153 & First branch height & $30 \mathrm{~cm}$ \\
\cline { 2 - 3 } 154 & Circumference at ground level & $35.4 \mathrm{~cm}$ \\
\cline { 2 - 3 } 155 & Circumference at first branch & $27.7 \mathrm{~cm}$ \\
\cline { 2 - 3 } & Radius of the canopy & $141 \mathrm{~cm}$ \\
\cline { 2 - 3 } 156 & Graft union appearance & Smooth and not visible \\
\cline { 2 - 3 } & &
\end{tabular}

157 Although, flower was formed, it did not develop fruit. This is not unexpected because baobab is

158 known to be self-incompatible (Venter et al, 2017). There is expectation of more flowers and 159 fruiting would occur after few months. 


\section{Conclusion}

164 The studies ongoing has shown that there is great possibility of drastically reducing the maturity

165 period of baobab to make it attractive to farmers to go into its cultivation. Although, cultural

166 practices were not the best for our studies, anthesis occurred earlier than ever reported for baobab.

167 Further investigations are therefore going on at Ho Technical University (HTU) to lead to its

168 domestication.

\section{Acknowledgement}

170 The authors appreciate the support given by the Vic-Chancellor of HTU (Professor Ben Q.

171 Honyenuga) and the University management. We also thank both teaching and non-teaching staff

172 of the Department of Agro Enterprise Development of HTU as well as students of the department

173 who are working in one way or the other on the baobab project. We also thank the whole HTU

174 community for celebrating our progress.

\section{References}

177 - Agbohessou M, Salako SV, Idohou R, Gbedomon RC, Hounkpevi A, Chadera FJ, 178 Glelekakai R and Assogbadjo AE. Status of vegetative propagation of baobab: a review.

$179 \quad$ African crop science journal. 2020; 28: 215-224. different grafting methods for vegetative propagation of baobab (Adansonia digitata L.) in Kenya to assist its domestication and promote cultivation. Forests, Trees and Livelihoods. 2017; (26): 2, 85-95. 
- El-Bably SMZ and Rashed NM. Influence of pre germination treatments on overcoming seed dormancy and seedling growth of baobab (Adansinia digitata L.). Zagazig J of Agric Res. 2018; 45 (2): 465-476.

- Gebauer J, Adam YO, Sanchez, AC, Darr, D et al. Africa's wooden elephant: the baobab tree (Adansonia digitata L.) in Sudan and Kenya: a review. Genet Resource Crop Evol. 2016; 63:377-399.

- Jamnadass RH, Dawson IK, Franzel S, Leakey RRB, Mithofer D, Akinnifesi FK and Tchoundjeu Z. Improving livelihood and nutrition in sub-Saharan Africa through the promotion of indigenous and exotic fruit production in smallholders' agroforestry systems: a review. International forestry review. 2011; 13 (3): 338-354.

- Jensen JS, Bayala J, Sanou H, Korbo A, Raebild A, Kambou S, Tougiani A, Bouda H, Larsen AS and Parkouda C. A research approach supporting domestication of baobab (Adansonia digitata L) in West Africa. New Forests. 2011; (41):317-335.

- Lautenschlager T, Teutloff N, Gunther M, Neinhuis C. Adansonia digitata germination tests. Elephants or heat: what causes scarification of seed to facilitate germination. Botanical studies. 2020; 61:19 DOI: 10.1186/s40529-020-00296-0

- Mbora A, Jamnadass R and Lilleso JPB. Growing high priority fruits and nuts in Kenya: uses and management. Nairobi. The world agroforestry centre. 61 pp. ISBN: 978-929059-236-5. 2008.

- North R, Darr D, Gebauer J, Mithofer D, Owino WO, Rimberia FK, Kehlenbeck K, Siddig E et al. Promoting the use of baobab (Adansonia digiatata $\mathrm{L}$ ) in rural communities in Eastern Africa. Conference on international research on food security, natural resource 
management and rural development. organized by Czech University of Life Sciences Prague. 2014.

- Sidibe M and Williams JT. Baobab Adasonia digitata. International Centre for Underutilized Crops, Southampton, UK. 2002.

- Simbo DJ, De Smedt S, Van de Blicke N, De Meulenaer B, Van Camp J, Uytterhoeven V, Tack F and Samson R. Opportunities for domesticating African baobab (Adansonia digitata L) multi-trait fruit selection. Agroforest syst. DOI: 10.1007/s10457-012-9568-7. 2012. Baobabs (Adansonia digitata L.) are self-incompatible and male trees can produce fruits if hand-pollinated. South African journal of botany. 109: 263-268. 2017. management. 2010; (261): 630-639. 


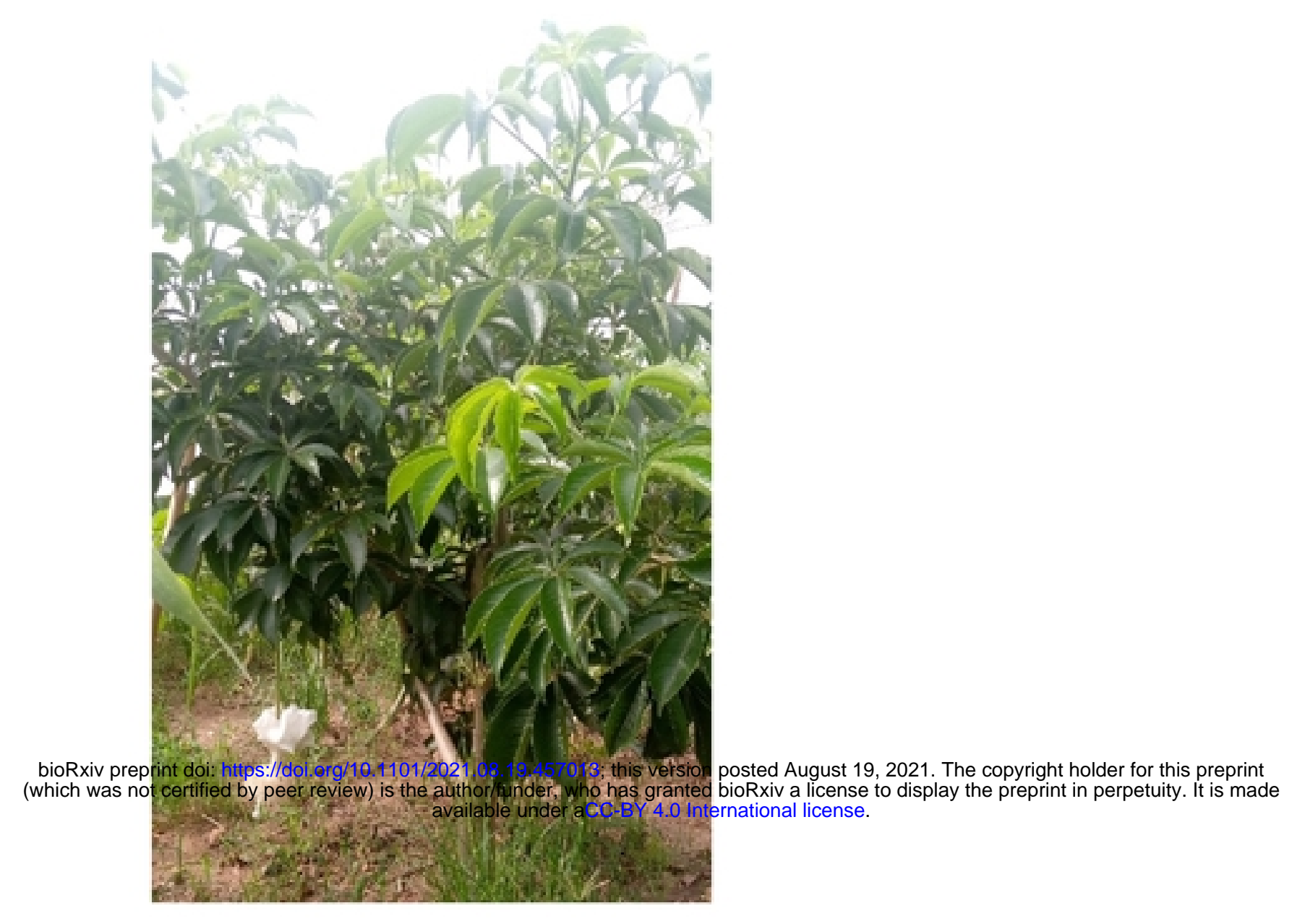

Figure 


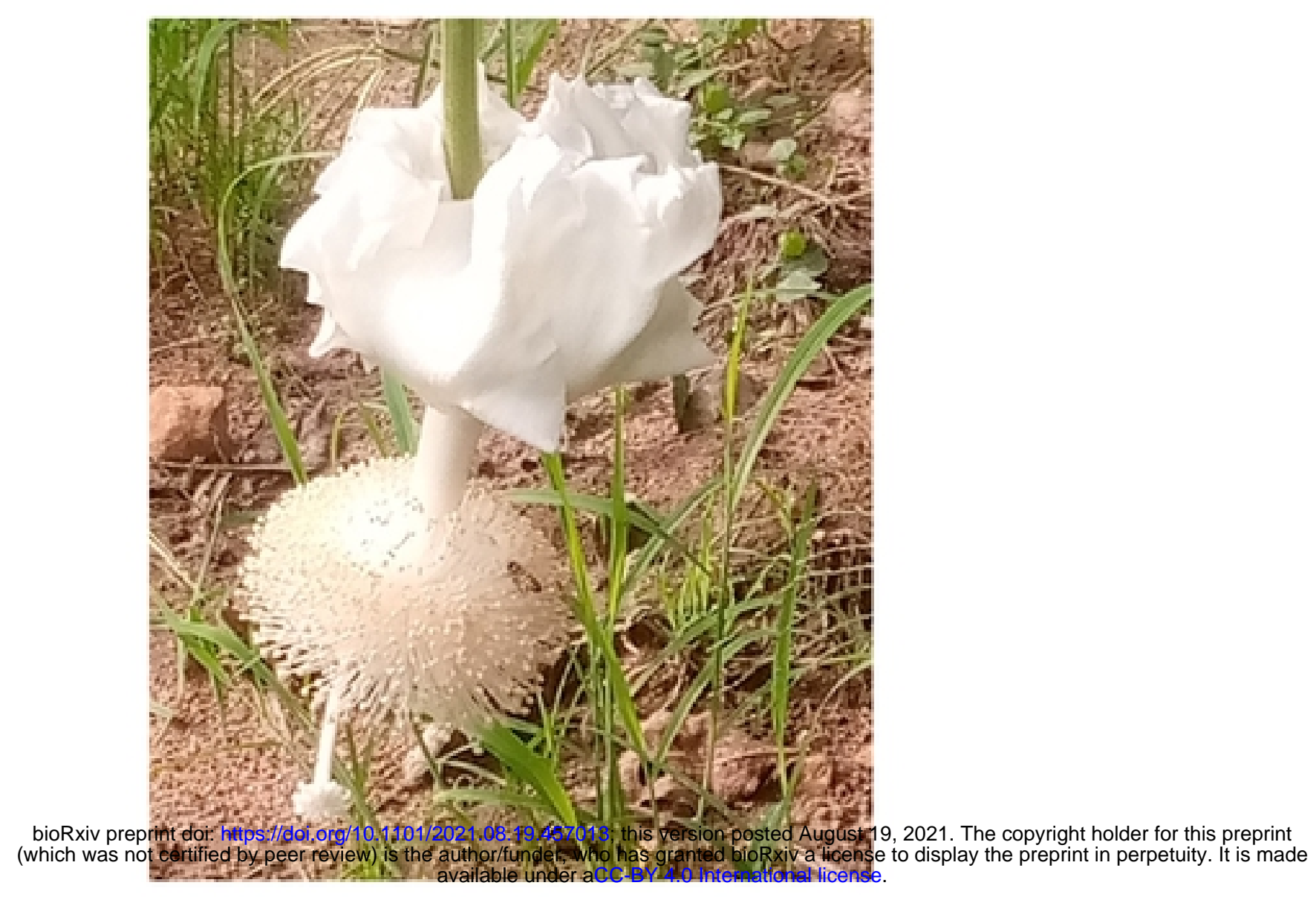

Figure 